\title{
Redescripción de la hembra de Lutzomyia vattierae (Diptera: Psychodidae, Phlebotominae) de la serranía de La Macarena, Colombia
}

\author{
Eduar Elías Bejarano1, Patricia Duque², Iván Darío Vélez² \\ 1 Grupo de Investigaciones Biomédicas, Universidad de Sucre, Sincelejo, Colombia. \\ 2 Programa de Estudio y Control de Enfermedades Tropicales-PECET, Universidad de Antioquia, Medellín, \\ Colombia.
}

Introducción. Las especies de Lutzomyia del subgénero Sciopemyia poseen una distribución restringida y son poco frecuentes en las recolectas de flebotomíneos, con la excepción de Lutzomyia sordellii, que se encuentra desde Costa Rica hasta Argentina. Hasta la fecha sólo dos de estas especies se han hallado en Colombia, Lutzomyia sordellii y Lutzomyia preclara. Objetivo. El propósito de este artículo es redescribir la hembra de Lutzomyia vattierae, especie del subgénero Sciopemyia que se registra por primera vez en el territorio nacional.

Materiales y métodos. El estudio se desarrolló en la serranía de La Macarena, departamento del Meta. Esta serranía se ubica entre la cuenca del Orinoco y del Amazonas, y es independiente de la cordillera de Los Andes. Los flebotomíneos se recolectaron durante dos noches consecutivas entre las 18:00 y 06:00 horas usando una trampa de luz tipo CDC instalada dentro de un bosque primario.

Resultados. Se obtuvieron cuatro hembras del género Lutzomyia, las cuales fueron identificadas como L. vattierae, que se diferencia de L. preclara por la presencia de papila en el flagelómero III y de $L$. sordellii, por la forma y longitud de las espermatecas y de los ductos espermáticos individuales.

Conclusión. El hallazgo de esta especie incrementa a tres el número de taxones del subgénero Sciopemyia registrados en Colombia, que ahora incluyen a L. sordellii, L. preclara y L. vattierae.

Palabras clave: Psychodidae/anatomía e histología, clasificación, Phlebotominae, Lutzomyia, Colombia.

Redescription of the female of Lutzomyia vattierae (Diptera: Psychodidae, Phlebotominae) from the serranía de La Macarena, central Colombia

Introduction. Lutzomyia species of the subgenus Sciopemyia show restricted distribution and are rarely represented in sand fly captures, with the exception of $L$. sordellii, which is found from Costa Rica to Argentina. To date, only two of these species. L. sordellii and L. preclara have been reported in Colombia.

Objective. The female of $L$. vattierae was redescribed and the record of this Sciopemyia species established for in the Colombian national park, serranía de La Macarena.

Materials and methods. The study was carried out in the Serrania de La Macarena, in western province of Meta. The serrania is a small mountain range situated between the Orinoco and Amazon River basins and geographically separated from the Andes. Sand flies were collected during two consecutive nights with a CDC light trap placed from 18:00 to 06:00 hours in a primary forest.

Results. Four females belonging to the subgenus Sciopemyia were identified as L. vattierae, which differs from $L$. preclara by the presence of papillae on the third flagellomere and from $L$. sordellii by the form and length of the spermathecae and individual sperm ducts.

Conclusion. The number of known species of Sciopemyia in Colombia is now three and include L. sordellii, L. preclara, and L. vattierae.

Key words: Psychodidae, anatomy and histology, classification, Phlebotominae, Lutzomyia, Colombia. 
Los flebotomíneos del subgénero Sciopemyia Barretto, 1962, considerado como género Sciopemyia por Galati (1), se caracterizan por tener cabeza ancha con ojos pequeños, flagelómero I muy largo, labro-epifaringe corto, vena $\beta$ más corta que la mitad de $\alpha$ y palpómero quinto de menor longitud que la suma del tercero y cuarto (2). La hembra presenta espermateca tubular con estrías transversales, ductos individuales comúnmente lisos y largos, y armadura cibarial compuesta por cuatro dientes horizontales no equidistantes con pocos dientes verticales. El macho posee cuatro espinas en el estilo, coxita con o sin tufo de setas y carece de seta subterminal. Su parámero es simple, con una pequeña protuberancia dorsal o con setas modificadas.

De acuerdo con Young y Duncan (2), el subgénero incluye ocho especies, Lutzomyia sordellii (Shannon y Del Ponte, 1927), Lutzomyia microps (Mangabeira, 1942), Lutzomyia fluviatilis (Floch y Abonnenc, 1944), Lutzomyia servulolimai (Damasceno y Causey, 1945), Lutzomyia pennyi Arias y Freitas, 1981, Lutzomyia nematoducta Young y Arias, 1984, L. preclara Young y Arias, 1984, y L. vattierae Le Pont y Desjeux, 1992. En años recientes se describió también a Lutzomyia manciola (Ibanez-Bernal, 2001), que comparte algunas características con este subgénero (3), aunque su posición taxonómica definitiva continúa siendo incierta (1). La mayoría de las especies de Sciopemyia es poco frecuente en las recolectas de flebotomíneos, con la excepción de $L$. sordellii que aparece bien representada (4). El propósito de este artículo es redescribir la hembra de $L$. vattierae, especie flebotomínea del mencionado subgénero, que se registra por primera vez en Colombia.

\section{Materiales y métodos}

\section{Área de estudio}

Los flebotomíneos se recolectaron en Los Andes,

\footnotetext{
Correspondencia:

Eduar E. Bejarano, Centro de Diagnóstico Médico, Universidad de Sucre, Carrera 14 No. 16 B-32, Apartado Aéreo 406, Sincelejo, Colombia.

Teléfono: (575) 282 0830; Fax: (575) 2821240.

eduarelias@yahoo.com

Recibido: 21/06/06; aceptado: 07/09/06
}

municipio de La Macarena ( $\left.02^{\circ} 11^{\prime} \mathrm{N}, 73^{\circ} 47^{\prime} \mathrm{O}\right)$, departamento del Meta, Colombia. Este municipio posee una temperatura media de $27^{\circ} \mathrm{C}$ y una precipitación promedio anual de $2.399 \mathrm{~mm}$ (5). Su territorio es montañoso, sobresaliendo al norte de la cabecera municipal la serranía de La Macarena con elevaciones de hasta $2.000 \mathrm{msnm}$. Esta serranía, cuyas rocas alcanzan los 1.200 millones de años de antigüedad, es una de las formaciones más antiguas del planeta, por lo tanto, se considera que los organismos que la habitan tienen una larga historia evolutiva, y que ha producido especies endémicas adaptadas a las condiciones particulares de la serranía (6). Geográficamente, se encuentra ubicada entre la cuenca del Orinoco y el Amazonas, es independiente de la Cordillera de Los Andes y aparece como una prolongación hacia el occidente del escudo guyanés.

\section{Recolección e identificación de flebotomíneos}

La recolección de los especímenes se desarrolló el 30 de noviembre y 1 de diciembre de 1994, usando una trampa de luz tipo CDC (Communicable Disease Center) entre las 18:00 y 06:00 horas, la cual se instaló dentro de un bosque primario a una altura de 1,5 m. Los flebotomíneos se aclararon en lactofenol, se montaron en bálsamo de Canadá y se identificaron con la clave taxonómica de Galati (7) y las descripciones originales de algunas especies. Las ilustraciones se realizaron con una cámara lúcida Nikon 1,25 X y las estimaciones morfométricas con un micrómetro ocular adaptado a un microscopio Nikon YS2-T. Los ejemplares recolectados están depositados en la "Colección de Vectores y Hospedadores Intermediarios de Enfermedades Tropicales" (VHET) del Programa de Estudio y Control de Enfermedades Tropicales (PECET) de la Universidad de Antioquia en Medellín, Colombia.

\section{Resultados}

Se recolectaron cuatro hembras del género Lutzomyia, las cuales fueron identificadas como L. vattierae (figuras $1 \mathrm{~A}-1 \mathrm{G}$ ). A continuación se redescribe la hembra de esta especie, haciendo énfasis en los caracteres morfológicos que no fueron detallados en la descripción original de Le Pont y Desjeux (8). El rango de variación morfométrica se expresa en micras entre paréntesis. 


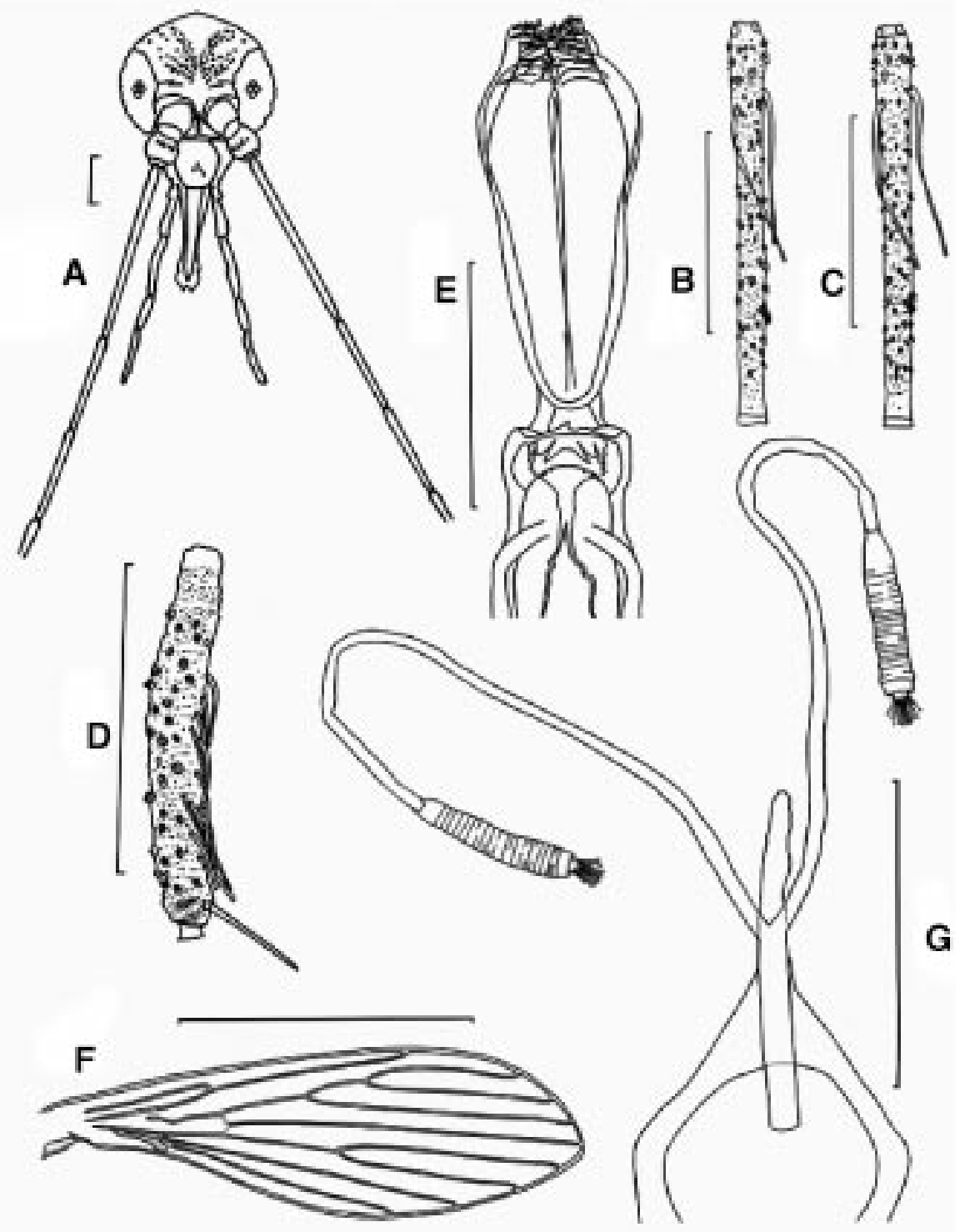

Figura 1. Lutzomyia vattierae Le Pont y Desjeux, 1992, flebotomíneo recolectado en Los Andes, La Macarena, Meta, Colombia. A: cabeza, B: flagelómero II, C: Flagelómero III, D:palpómero 3, E: faringe y cibario; escala métrica = $100 \mathrm{~mm}$. F: ala, escala métrica $=1.000 \mathrm{um}$. G: espermatecas; escala métrica $=100 \mathrm{um}$. 


\section{Lutzomyia (Sciopemyia) vattierae Le Pont y Desjeux, 1992}

Cabeza ancha que mide 322 (307-322), con una altura desde el vértex hasta el margen distal del clipeo de 365 (355-374) (figura 1A). Ojos pequeños, con una longitud de 180 (178-186) y anchura de 89 (80-90). Distancia interocular 142 (127-145). Sutura interocular incompleta. Longitud del clípeo 106 (106-110). Longitud del labro-epifaringe 190 (187-190). Lacinia maxilar con alrededor de ocho dientes longitudinales externos y 19 dientes internos. Hipofaringe con aproximadamente 10 dientes apicolaterales escasamente desarrollados. Suturas labiales que forman una furca. Longitud de los flagelómeros: I, 371 (371-406), II, 205 (199-215) y III, 197 (185-200). El flagelómero I es más largo que el labro, alcanzando la mitad proximal del quinto palpómero. Papilas sobre los flagelómeros I al III y IX al XIV, la del flagelómero II insertada en el tercio distal del borde interno (figura 1B y 1C). Ascoides pareados y simples en los flagelómeros I al XIII, sin sobrepasar el ápice de cada segmento. Flagelómero II con ascoide interno implantado de forma más proximal que el externo, ambos sin alcanzar el nivel de la papila. Longitud de los palpómeros: primero, 45 (44-47), segundo, 70 (63-72), tercero, 130 (122132), cuarto, 70 (64-70) y quinto, 120 (103-125). Fórmula palpal: 1.(2.4).5.3. o 1.2.4.(3.5). Palpómero tercero con una espina preapical y con espinas de Newstead de ápice no dilatado distribuidas entre los 0,29 y 0,80 proximales del segmento (figura 1D). Faringe con finas estrías transversales entre los 0,85 y 0,94 distales (figura $1 \mathrm{E}$ ). Adicionalmente, presenta pequeñas espinas dispuestas en filas paralelas a partir de los 0,95 distales, y armadura cibarial formada por cuatro dientes horizontales puntiagudos inclinados hacia adentro, con un amplio espacio entre el par medio donde se proyecta una estructura quitinosa (figura 1E). Además, cuenta con cerca de siete dientes verticales atrofiados y pequeños dientes postero-laterales triangulares flanqueando los dientes horizontales. El área pigmentada es muy tenue y el arco cibarial es incompleto en la parte media.

Escudo, pósnoto, catepisterno, catepimero, metaepisterno y coxas de color amarillo oscuro. Pleura con 24 (19-27) setas anepisternales superiores y una (1-2) inferior. Sensilia ventrocer-vical ausente. Longitud del ala desde el inicio de la vena costa, $1.862(1.832-1.886)$ y anchura máxima, 576 (538$600)$. La longitud de las venas principales del ala (figura 1F) es: $\alpha 526$ (458-526), $\beta 214$ (214-254), $\gamma 288$ (259-288), $\delta 144$ (86-144), $\varepsilon 643$ (593-643), $\theta 912$ (893-914), $\pi 122$ (92-122) y $R_{5} 1.219$ (11901229); d y $p$ son positivos. Espermatecas tubulares, con finas estrías transversales y un segmento apical de mayor tamaño que los 17-20 anillos precedentes (figura $1 \mathrm{G}$ ). Longitud de la espermateca, 53 (50-56), anchura máxima, 12 (1012). Botón terminal grande con filamentos largos. Ductos individuales largos y lisos. Longitud de los ductos individuales, 207 (195-238), anchura, 5 (5). Ducto común con paredes lisas. Longitud del ducto común 66 (62-66), anchura, 9 (9).

\section{Discusión}

Las especies del subgénero Sciopemyia se caracterizan por ser poco antropofílicas $(2,8)$, lo que coincide con la captura de $L$. vattierae exclusivamente con trampas de luz tipo CDC en la serranía de La Macarena, a pesar de que paralelamente a este trabajo se desarrollaron otros estudios usando cebo humano (Bejarano EE, datos sin publicar). Los ejemplares de $L$. vattierae examinados aquí concuerdan con la descripción original de la especie realizada por Le Pont y Desjeux (8), salvo por presentar los cuatro dientes horizontales más desarrollados que se proyectan por encima del área quitinosa (figura 1E).

Entre los taxones que conforman este subgénero, $L$. vattierae se asemeja especialmente a $L$. servulolimai y $L$. microps, de las cuales se diferencia por exhibir papila sobre el flagelómero III. Esta estructura sensorial también permite separarla de todas las demás especies de Sciopemyia, con la excepción de L. sordellii. De esta última se distingue por la mayor longitud y forma tubular de las espermatecas, dado que $L$. sordellii presenta espermatecas cortas y dilatadas en su mitad apical. Adicionalmente, los ductos espermáticos individuales de $L$. vattierae son extensos, delgados y totalmente lisos, mientras los de L. sordellii son cortos, gruesos y cubiertos por sutiles estrías semejantes a puntos. 
L. vattierae se diferencia además de L. fluviatilis y $L$. preclara por tener un ducto común mucho más largo, y de L. nematoducta por la longitud y anchura de los ductos individuales. A lo largo de su extensión, los ductos de $L$. vattierae muestran un mismo grosor, con un radio de la longitud del ducto individual/espermateca de 3,91, en tanto que en $L$. nematoducta la anchura de los ductos varía a lo largo de éstos y el radio de la longitud del ducto individual/espermateca es 13,3 (9). De otro lado, $L$. vattierae se distingue fácilmente de L. manciola por exhibir ductos espermáticos lisos y botón terminal de la espermateca sin lóbulos.

La ubicación de $L$. manciola dentro del subgénero Sciopemyia se justificaría por presentar el flagelómero I largo, palpómero quinto relativamente corto, arco cibarial incompleto, faringe sin espinas, espermatecas con anulaciones y ducto espermático común más corto que los ductos individuales. Sin embargo, tal como lo menciona lbáñez-Bernal (3), el botón terminal lobulado de la espermateca, las estrías de los ductos espermáticos y el tamaño y la disposición de los dientes verticales y horizontales no soportan su inserción en este grupo taxonómico.

El presente trabajo constituye el primer registro de la presencia de $L$. vattierae en Colombia, donde previamente se había documentado el hallazgo de L. sordellii, L. preclara y L. sp de Vichada (10), esta última considerada por Young y Morales (11) como un sinónimo junior de L. preclara. Es importante tener en cuenta que estas especies se han encontrado con anterioridad en el departamento del Meta (12) y que si bien los machos de $L$. preclara pueden separase con facilidad de los de $L$. vattierae por poseer un tufo de setas en la coxita, los machos de L. sordellii y L. vattierae, por el contrario, son morfológicamente muy similares. Su diferenciación sólo es posible empleando algunos caracteres morfométricos de la cabeza y la terminalia, lo que denota la relevancia que tiene la hembra para la correcta determinación taxonómica de estas especies.

La distribución geográfica de L. vattierae se encuentra restringida a los países andinos de Bolivia y Perú $(8,13)$. Su presencia en la serranía de La Macarena en Colombia comprueba la extraordinaria riqueza biológica de este aislamiento montañoso, sumándose al hallazgo previo, por primera vez en el país, de Brumptomyia pintoi (Costa Lima, 1932) $(14,15)$. No obstante, se necesitan más investigaciones para ampliar el aún escaso conocimiento de la fauna flebotomínea que alberga esta región.

\section{Conflicto de intereses}

Los autores declaran que no existe conflicto de intereses alguno en torno al estudio publicado.

\section{Financiación}

Este trabajo fue financiado por el Programa de Estudio y Control de Enfermedades Tropicales, PECET, de la Universidad de Antioquia.

\section{Referencias}

1. Galati EA. Classificação de Phlebotominae. En: Rangel EF, Lainson R, editores. Flebotomíneos do Brasil. Rio do Janeiro: Editora Fiocruz; 2003. p.23-51.

2. Young DG, Duncan MA. Guide to the identification and geographic distribution of Lutzomyia sand flies in Mexico, the West Indies, Central and South America (Diptera: Psychodidae). Mem Amer Ent Inst 1994;54:1881.

3. Ibáñez-Bernal S. Notes on the Psychodidae (Diptera) of Belize: Subfamilies Bruchomyiinae and Phlebotominae. Ann Entomol Soc Am 2001;94:367-85.

4. Galati EA, Nunes VLB, Rego Jr FA, Oshiro ET, Chang MR. Estudo de flebotomíneos (Diptera: Psychodidae) em foco de leishmaniose visceral no Estado de Mato Grosso do Sul, Brasil. Rev Saúde Pública 1997;31:378-90.

5. Instituto Geográfico Agustín Codazzi. Diccionario geográfico de Colombia. $3^{\underline{a}}$ ed. Santafé de Bogotá: Instituto Geográfico Agustín Codazzi; 1996.

6. Mejía CA. Fauna de la Serranía de La Macarena. Santafé de Bogotá: Ediciones Uniandes \& Amazonas Editores; 1995.

7. Galati EAB. Morfologia, terminologia de adultos e identificação dos táxons da América. En: Rangel EF, Lainson R, editores. Flebotomíneos do Brasil. Rio do Janeiro: Editora Fiocruz; 2003. p.53-175.

8. Le Pont F, Desjeux P. Phlébotomes de Bolivie. Description de Lutzomyia vattierae n. sp. et de la femelle de L. antezanai Le Pont, Dujardin et Desjeux, 1989 (Diptera, Psychodidae). Bull Soc Ent Fr 1992;97:2638.

9. Andrade Filho JD, Galati EA, de Sousa RN, Falcão AL. Description of the female of Sciopemyia servulolimai 
(Damasceno \& Causey) (Diptera: Psychodidae). Mem Inst Oswaldo Cruz 2003;98:503-5.

10. Young DG, Arias JR. The microps group of Lutzomyia França with descriptions of two new species from South America (Diptera, Psychodidae, Phlebotominae). Mem Inst Oswaldo Cruz 1984;79:425-31.

11. Young DG, Morales A. New species and records of phlebotomine sand flies from Colombia (Diptera: Psychodidae). J Med Entomol 1987;24:651-65.

12. Montoya-Lerma J, Ferro C. Flebótomos (Diptera: Psychodidae) de Colombia. En: Amat G, Andrade MG, Fernández $F$, editores. Insectos de Colombia. Volúmen II. Colección Jorge Alvárez Lleras, No. 13. Academia
Colombiana de Ciencias Exactas, Físicas y Naturales. Santafé de Bogotá: Centro Editorial Javeriano; 1999. p.211-45.

13. Ogusuku E, Guevara Z, Revilla L, Inga A, Pérez JE. Descripción de Lutzomyia pisuquia sp. n. (Diptera: Psychodidae), y dos registros nuevos de Lutzomyia para el Perú. Rev Peru Entomol 2001;42:21-5.

14. Bejarano EE, Duque $P$, Vélez ID. Primer registro del flebotomíneo Brumptomyia pintoi (Diptera: Psychodidae) en Colombia. Caldasia 2004;26:311-4.

15. Bejarano EE. Lista actualizada de los psicódidos (Diptera: Psychodidae) de Colombia. Folia Entomol Mex 2006;45:47-56.

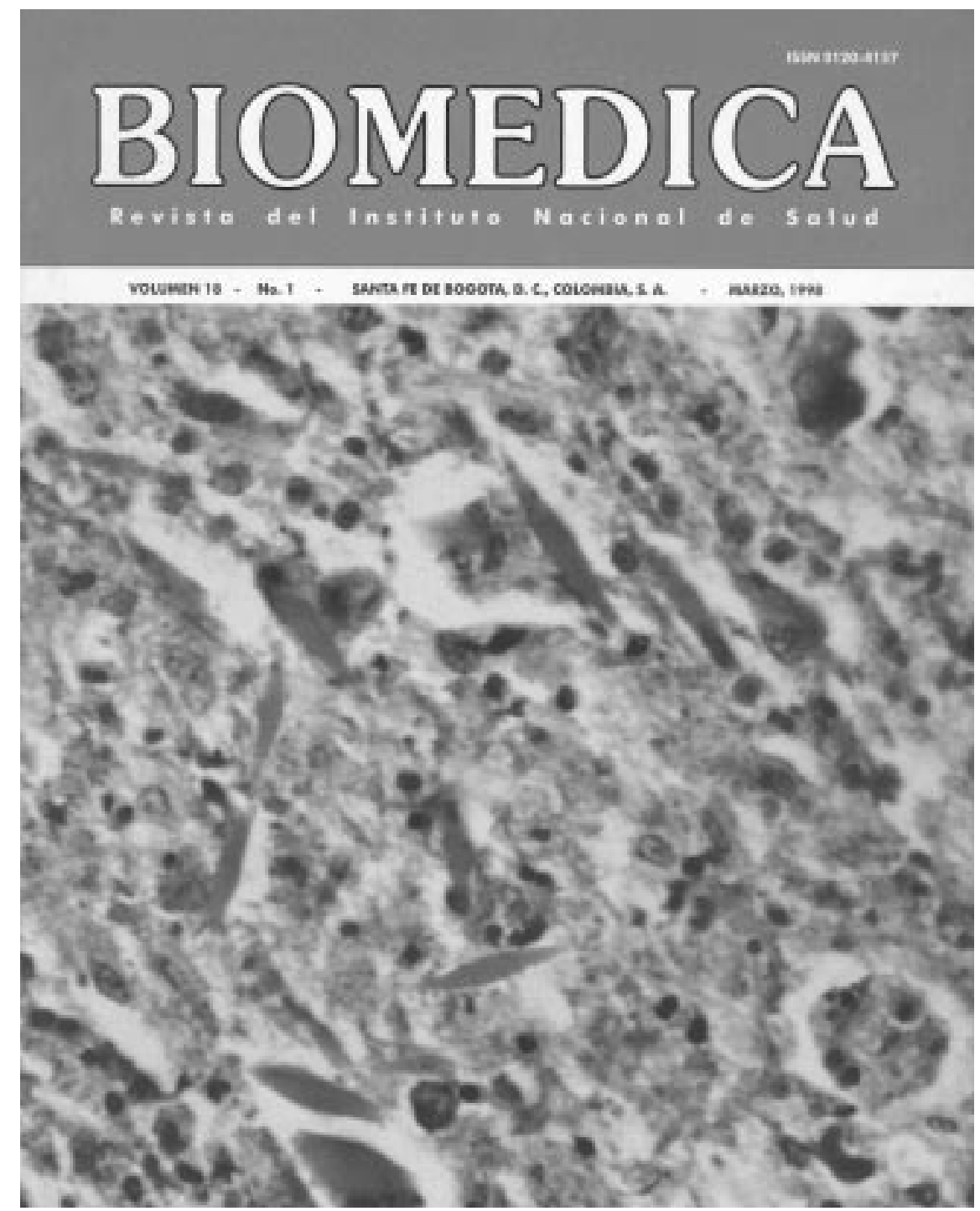

